\title{
On the Burning of Single Drops of Fuel in an Oxidizing Atmosphere
}

\author{
M. GOLDSMITH ${ }^{2}$ and S. S. PENNER ${ }^{3}$
}

Daniel and Florence Guggenheim Jet Propulsion Center, California Institute of Technology, Pasadena, Calif.

\begin{abstract}
A simplified model for the process of steady burning of a stationary droplet of fuel in an oxidixing atmosphere has been examined. Explicit expressions have been obtained for the burning rate of the fuel droplet, for the temperature at the flame front, and for the radius of the combustion surface. The principal assumptions on which our analysis is based are: the flame front is established at a spherical surface surrounding the drop; the rates of delivery of fuel and oxygen to this surface are in stoichiometric proportions; the rates of reaction at the flame front are fast compared to the rates of delivery of combustible gases. Our analysis is an extension and generalization of the work of G. A. E. Godsave. We are able to delete several of Godsave's restrictive assumptions by use of an efficient method for formulating the problem in which only integrated forms appear for the expressions of conservation of mass and energy. Our theoretical formulas provide a satisfactory correlation of Godsave's experimental results.
\end{abstract}

$$
\begin{aligned}
& \text { Nomenclature } \\
& r \quad=\text { radial distance from center of drop } \\
& T=\text { temperature } \\
& T^{*} \quad=\text { standard reference temperature (usually } 298.16 \mathrm{~K} \text { ) } \\
& t \quad=\text { time } \\
& \rho \quad=\text { density of gas mixture } \\
& \rho_{l}=\text { density of liquid fuel } \\
& \left(c_{p}\right)_{K}=\text { specific heat at constant pressure of gaseous species } K \\
& \mathrm{Cl}_{l} \quad=\text { specific heat of liquid fuel } \\
& \lambda=\text { thermal conductivity } \\
& \Delta l \quad=\text { specific latent heat of vaporization of fuel } \\
& D_{K} \quad=\text { binary diffusion coefficient for species } K \\
& Y_{K} \quad=\text { weight fraction of species } K \text { in gaseous mixture } \\
& \dot{m}_{F} \quad=\text { mass, rate of flow of fuel vapor } \\
& \left(h_{K}\right)_{T}=\text { specific enthalpy of species } K \text { at temperature } T \\
& \gamma_{K}=\dot{m}_{K} / \dot{m}_{F} \text {, ratio of mass rate of flow of species } K \text { to the }
\end{aligned}
$$

Subscripts

$O=$ oxidizer $\quad F=$ fuel vapor $\quad I=$ inertgas

$o=$ condition in ambient gas $(r \rightarrow \infty)$

$c=$ condition at combustion zone

$l=$ condition at drop surface

Received March 8.1954.

${ }^{1}$ Supported by the O.O.R. under Contract DA 04-495-Ord-446, Dept. of the Army Project No. 599-01-004, Ordnance Research and Development Project No. TB 2-001, OOR Project No. 834. The authors are happy to express their appreciation to Dr. H. S. Tsien for helpful discussions.

${ }^{2}$ Daniel and Florence Guggenheim Jet Propulsion Fellow. Stud. Mem. ARS.

${ }_{3}$ Associate Professor of Jet Propulsion.

\section{Introduction}

$\mathbf{H}$ ETEROGENEOUS combustion is of importance in such widely different applications as stationary boilers, diesel engines, gas turbines, and rocket motors. In spite of the diversity of these machines, the combustion problems involved in their design bear marks of similarity. Numerous articles relating to heterogeneous combustion have been published, some of which are listed in the bibliography (1). ${ }^{4}$

The present preliminary theoretical investigations are restricted to the burning of single drops of fuel in an oxidizing atmosphere. Our theoretical results are used to correlate some experimental studies carried out by G. A. E. Godsave $(2,3)$. In technical combustion processes with little interference between burning droplets, the single-drop analysis may be a useful first approximation.

Experimental data on the burning of single drops of fuel have been published by Godsave (3) and Topps (4). Topps (4) studied the rate of burning of small fuel drops falling through a heated oxidizing atmosphere. Godsave (3) suspended small droplets on a fine quartz fiber and examined the burning droplet and the flame front as a function of time. In this way quantitative data were obtained for the rate of burning of the suspended droplet. Gadsave $(2,5)$ obtained a successful interpretation of his results on the assumption that the chemical reaction rates do not control the rate of burning. This hypothesis simplifies the analytical treatment considerably.

Following Godsave (2), Spalding (6), and others, we postulate the following mechanism for the combustion processes: oxidizer is delivered from the surrounding atmosphere to the region of active combustion by convection and diffusion; the fuel evaporates and diffuses, without chemical change, to the reaction front, which is assumed to be a spherical shell surrounding the droplet. The location of the reaction front is defined by the condition that the ratio of the mass rate of delivery of fuel to oxidizer corresponds to stoichiometric proportions. It is assumed that the reactants are consumed instantaneously upon reaching the flame front. The problem of determining the rate of burning therefore reduces to finding the solutions of the appropriate transfer problems. Because it is to be expected that the rates of mass and heat transfer will be increased by the effects of convection, a lower limit for the burning rate will be obtained if the analysis is made for a droplet burning in a still atmosphere, by neglecting the convection of hot gases over the fuel droplet. Although fuel may be injected into the combustion chamber of an engine at a velocity appreciably different from the gas velocity in the

\footnotetext{
${ }^{4}$ Numbers in parentheses refer to References on page 251 .

We have recently learned that extensive work on combustion and evaporation has been carried out in Japan in recent years. Some of the important papers are the following: "On the Evaporation Velocity of a Liquid Droplet in a High Temperature Gas," by $\mathrm{Y}$. Tanasawa and K. Kobayashi, the Technology Reports of the Tohoku University, vol. XIV no, 2, 1950. "Experimental Study on Evaporation and Combustion of Fuel Droplet," by $\mathbf{S}$. Kumagai and H. Isoda, Science of Machine, vol. 4, 1952, pp. 337342. "Combustion of Fuel Droplets," by $\mathrm{S}$. Kumagai and $\mathrm{A}$. Kimura. Science of Machine, vol. 3, 1951, pp. 431-434.
} 
chamber, it appears likely that, in some cases, the droplet is slowed down rapidly to the local gas velocity because of aerodynamic drag.

In the following Section II a theory for the burning of a droplet in a still atmosphere will be presented. The comparison of our theoretical results with the experimental studies of Godsave (3) is carried out in Section III. Our method of analysis differs from that of Godsave in that we utilize only integrated forms for the energy and continuity equations, thereby simplifying the analytical treatment, since only firstorder differential equations occur. Because of the improvement in the method of formulating the problem, we are able to derive without difficulty an explicit expression for the mass rate of fuel flow without introducing the invalid approximations that the thermal conductivity and the specific heat of fuel vapor are independent of the temperature. Furthermore, we extend Godsave's analysis in two important respects by obtaining explicit expressions for (a) the temperature of the combustion surface, and (b) the radius of the combustion surface.

\section{A Simplified Model for the Burning of Single Drops of Fuel}

For the sake of clarity we tabulate all of the important assumptions upon which our analysis is based. These are: 1 The droplets are spherical. 2 Convection effects may be neglected. 3 The flame front surrounding the drop is represented by a spherical surface concentric with the drop. All reactions take place instantaneously at this surface, at which the delivery rates of fuel and oxidizer are in stoichiometric proportions. 4 Steady-state conditions are assumed for fixed droplet sizes. This restriction greatly facilitates the mathematical treatment. It is reasonable to assume that the solution obtained for a fixed size applies to a drop decreasing in size when it reaches the radius used in the steadystate solution. 5 The effect of heat transfer by radiation is neglected. 6 Mean values will be used, when appropriate, for the physical properties. 7 The temperature of the liquid drop is assumed to be uniform and equal to the boiling temperature. Although this assumption is questionable (7), it does not exert a large effect on the theoretical results. 8 The pressure is assumed to be uniform throughout the system.

A schematic diagram of an evaporating and burning fuel droplet in an oxidizing atmosphere is shown in Fig. 1. The radius of the liquid drop is $r_{l}$ and its temperature is the normal boiling point $T_{l}$. The radial distance of the combustion sur-

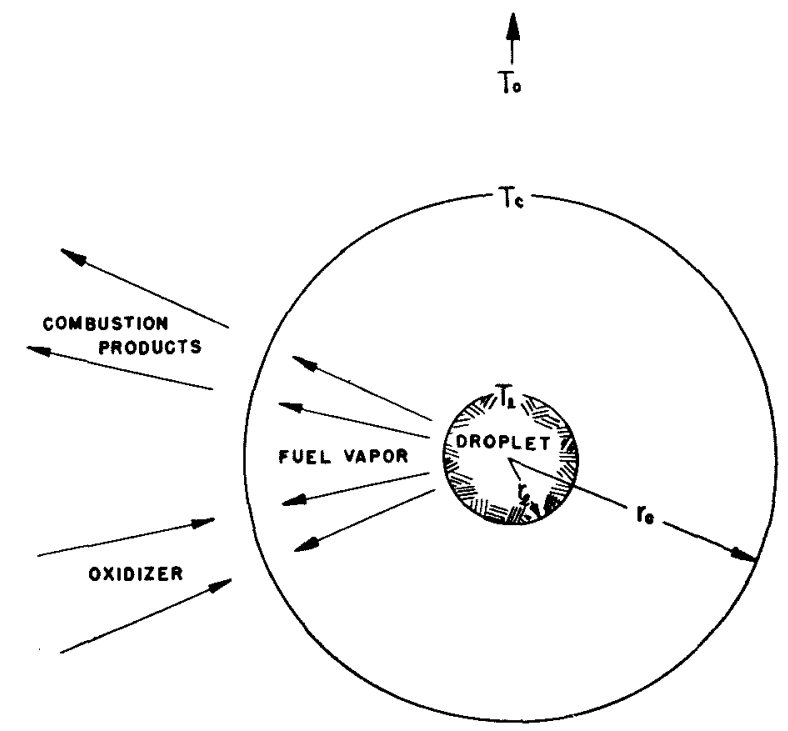

FIG. 1 SCHEMATIC DIAGRAM OF BURNING FUEL DROP

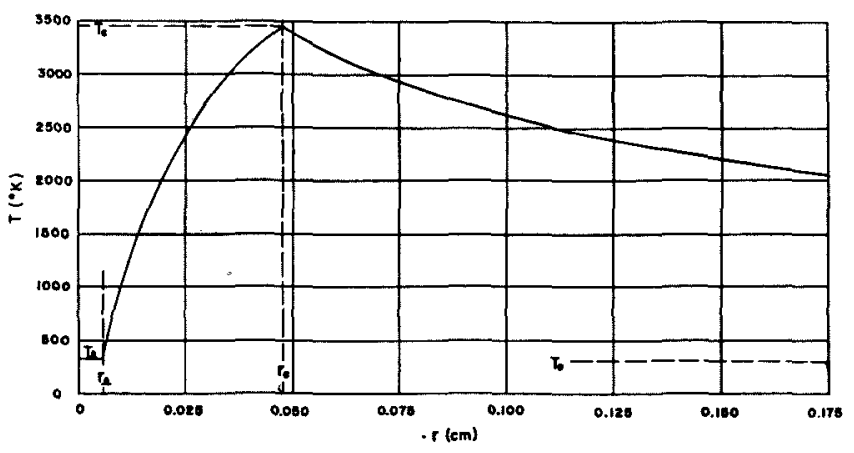

FIG. 2a TEMPERATURE ( $T$ ) AS A FUNCTION OF DISTANCE FROM CENTER OF DROP $(r)$ FOR 0.010 CM-DIAM BENZENE DROPLET BURNING IN AIR AT ATMOSPHERIC PRESSURE (UNCORRECTED FOR DISSOCIATION)

face from the center of the liquid droplet is $r_{c}$ and its temperature is $T_{c}$. The oxygen-inert gas mixture at a large distance from the combustion surface is at the temperature $T_{o}$. In order to clarify further our physical picture we show in Fig. 2a a plot of the temperature $T$ as a function of the radial distance $r$ from the center of the drop and, in Figs. $2 \mathrm{~b}$ to $2 \mathrm{~d}$, diagrams of the weight fractions of fuel $Y_{F}$, oxidizer $Y_{o}$, and inert gas $Y_{1}$ as a function of $r$. The data shown in Figs. 2a to $2 \mathrm{~d}$ correspond to the burning of a droplet of benzene in air for $r_{l}=0.005 \mathrm{~cm}$ and $T_{o}=300^{\circ} \mathrm{K}$ (compare Section III). The profile for the weight fraction of inert gas has been drawn on the assumption that the physical properties of combustion products and inert gas (in the oxidizer-inert gas mixture) are alike. If this assumption is not made, we must treat ternary gas mixtures both for $r<r_{c}$ and for $r>r_{c}$. This refinement can be introduced without difficulty but does not appear to be warranted in view of the crudeness of our model.

Let $\dot{m}_{F}$ represent the steady-state mass rate of fuel consumption, which is the desired eigenvalue of our boundaryvalue problem; $t$ is the time; $\rho, c_{p}$, and $\lambda$ represent, respectively, the density, specific heat at constant pressure, and thermal conductivity; $\Delta l$ equals the specific latent heat of evaporation of the fuel.

For a constant-pressure flow process, ${ }^{5}$ the first law of thermodynamics leads to the relation

$$
d h / d t=d q / d t
$$

where $d h / d t$ is the rate of enthalpy increase of the gases passing through a fixed volume to which the total rate of energy transfer is $d q / d t$. For a spherical shell bounded by the radii $r_{i}$ and $r_{f}$, the energy equation takes the following simple form

$$
\begin{gathered}
(d h / d t)_{f}-(d h / d t)_{i}=-\left\{\left[4 \pi r^{2} \lambda(d T / d r)\right]_{i}-\right. \\
\left.\left[4 \pi r^{2} \lambda(d T / d r)\right]_{f}\right\}
\end{gathered}
$$

Here the subscripts $i$ and $f$ identify, respectively, the surfaces at $r_{i}$ and $r_{f}$.

The general continuity equation for species $K$ can be written (8) in the form

$$
\dot{m}_{K}=4 \pi r^{2} \rho Y_{K}\left[\left(\dot{m}_{F} / 4 \pi r^{2} \rho\right)-\left(D_{K} / Y_{K}\right)\left(d Y_{K} / d r\right)\right] \ldots
$$

where $\dot{m}_{K}$ is the rate of mass transport of species $K, \rho$ is the density of the gas mixture, $Y_{K}$ equals the weight fraction of species $K$, and $D_{K}$ is the appropriate diffusion coefficient for species $K$. Equation [2] states that the total mass transport of species $K$ is equal to the sum of the mass transport of species $K$ associated with the movement of the average fluid, $Y_{K} \dot{m}_{F}$, and with mass transfer by diffusion, $-4 \pi r^{2} \rho D_{K}\left(d Y_{K} / d r\right)$. ${ }^{5}$ In our formulation we make no explicit use of the momentum
equation. It is easily shown that the condition for conservation
of momentum reduces to the statement that the pressure is
practically constant, which we assume to be the case in the analy- 


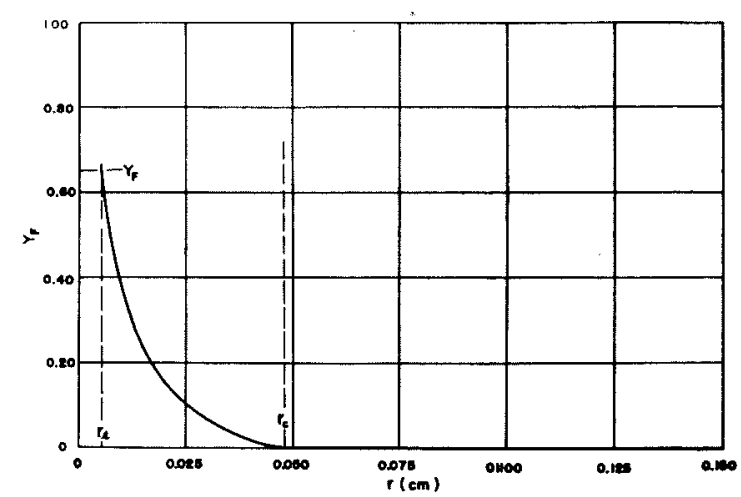

FIG. $2 b$ WEIGHT FRACTION OF FUEL VAPOR $\left(Y_{F}\right)$ AS FUNCTION OF DISTANCE FROM CENTER OF DROP $(r)$ FOR 0.010 CM-DIAM BENZENE DROPLET BURNING IN AIR AT ATMOSPHERIC PRESSURE

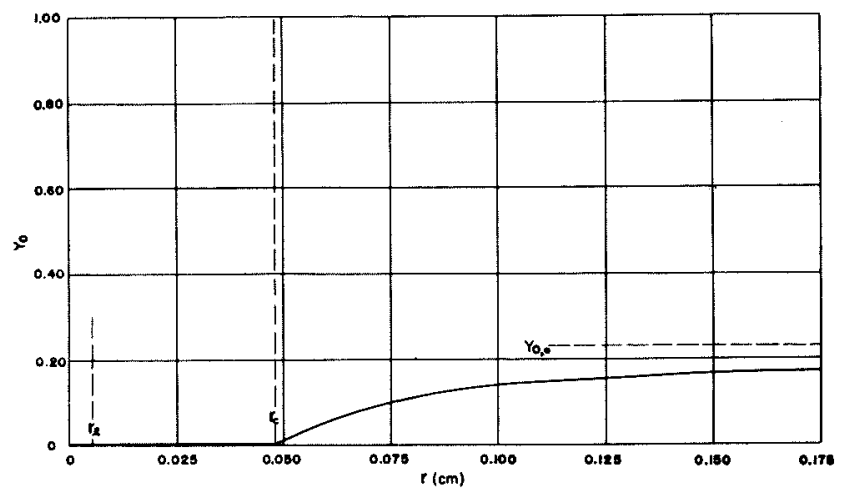

FIG. 2c WEIGHT FRACTION OF OXYGEN $\left(Y_{O}\right)$ AS FUNCTION OF DISTANCE FROM CENTER OF DROP $(r)$ FOR 0.010 CM-DIAM BENZENE DROPLET BURNING IN AIR AT ATMOSPHERIC PRESSURE

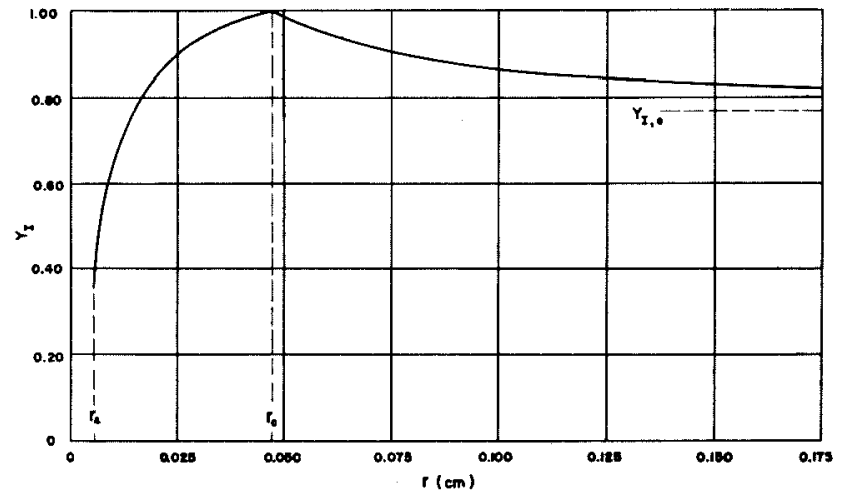

FIG. $2 \mathrm{~d}$ WEIGHT FRACTION OF INERT GAS $\left(Y_{I}\right)$ AS FUNCTION OF DISTANCE FROM CENTER OF DROP $(r)$ FOR 0.010 CM-DIAM BENZENE DROPLET BURNING IN AIR AT ONE ATMOSPHERE

\section{A Derivation of Godsave's (2) Equation for $\dot{m}_{F}$}

We apply the expression for conservation of energy, which is given in Equation [1], to the spherical shell between $r_{l}$ and $r$ for $r<r_{c}$. The rate of enthalpy transport at $r_{l}$ is

$$
\dot{m}_{F}\left(h_{F}\right)_{T l}
$$

and at $r$

$$
\dot{m}_{F}\left(h_{F}\right)_{T}
$$

The rate of energy transport by thermal conduction at $r_{l}$ is

$$
-\left[4 \pi r^{2} \lambda(d T / d r)\right] r_{l}=-\dot{m}_{F} \Delta l
$$

and the rate of energy transport at $r$ into the spherical shell between $r_{l}$ and $r$ is

$$
4 \pi r^{2} \lambda(d T / d r)
$$

Hence Equation [1] becomes

$$
\dot{m}_{F}\left[\left(h_{F}\right)_{T}-\left(h_{F}\right)_{T l}\right]=-\dot{m}_{F} \Delta l+4 \pi r^{2} \lambda(d T / d r)
$$

Or

$$
\mathbf{4} \pi r^{2}(d T / d r)=\left(\dot{m}_{F} / \lambda\right)\left[\Delta l+\int_{T_{l}}^{T}\left(c_{p}\right)_{F} d T\right]
$$

where the subscript $F$ to the specific heat identifies the fuel vapor. If it is assumed that $\lambda=\lambda_{1}$ is independent of temperature, and also that $\left(c_{p}\right)_{F}=\left(c_{p}\right)_{F 1}$ is constant, then Equation [3] becomes

$$
4 \pi r^{2}(d T / d r)=\left[\dot{m}_{F}\left(c_{p}\right)_{F 1} / \lambda_{1}\right]\left\{\left[\Delta l /\left(c_{p}\right)_{F 1}\right]+\left(T-T_{l}\right)\right\}
$$

Integration of the preceding expression between the limits $r=r_{l}$ at $T=T_{l}$ and $r=r_{c}$ at $T=T_{c}$ leads directly to Godsave's equation for $\dot{m}_{F}$, viz.,

$$
\dot{m}_{F}=\frac{4 \pi \lambda_{1}}{\left(c_{p}\right)_{F_{1}}} \frac{\ln \left\{1+\left[\left(c_{p}\right)_{F_{1}}\left(T_{c}-T_{l}\right) / \Delta l\right]\right\}}{\left[\left(1 / r_{l}\right)-\left(1 / r_{c}\right)\right]} .
$$

Reference to Equation [4] shows that for $r_{c} \gg r_{l}$, or for constant values of $r_{l} / r_{e}, \dot{m}_{F}$ is a linear function of the droplet radius. Furthermore, since $T_{c}$ is generally large compared to $T_{l}$, it follows that $\dot{m}_{F}$ is not a sensitive function of $T_{l}$.

It should be noted that Equation [4] was derived without making any special assumptions about the location of the reaction front. For this reason the expression for $\dot{m}_{F}$ contains two unknown parameters, $T_{c}$ and $r_{c}$. Godsave measured ${ }^{6} r_{c}$ and showed that for reasonable, assumed values of $T_{c}$, an acceptable correlation for the measured values of $\dot{m}_{F}$ was provided by Equation [4].

\section{B An Expression for $\dot{m}_{F}$ if $\lambda$ and $\left(c_{p}\right)_{F}$ are Linear Func- tions of the Temperature}

A refinement of Godsave's equation can be obtained without difficulty by deleting the assumptions (a) that $\lambda$ can be assigned an average value $\lambda_{1}$ in the temperature interval between $T_{l}$ and $T_{c}$, and $(b)$ that the specific heat of the fuel vapor is constant. Thus we write the following approximate expressions

$$
\lambda=\lambda_{l}\left(T / T_{l}\right) \ldots \ldots
$$

where $\lambda_{l}$ is the thermal conductivity of the fuel-inert gas mixture at the temperature $T_{l}$, and

$$
\left(c_{p}\right)_{F}=a+b T .
$$

where $a$ and $b$ are suitably chosen constants. Equations [3], [5], and [6] lead to the result

$$
\begin{aligned}
4 \pi r^{2}(d T / d r)=\left(\dot{m}_{F} T_{l} / \lambda_{l} T\right) & {\left[a\left(T-T_{l}\right)+\right.} \\
& \left.(b / 2)\left(T^{2}-T_{l}^{2}\right)+\Delta l\right]
\end{aligned}
$$

Integration of the preceding expression from $r_{l}, T_{l}$, to $r_{c}, T_{c}$ shows that

$$
\begin{aligned}
& \dot{m}_{F}=\frac{4 \pi \lambda_{l} r_{l}}{b T_{l}\left[1-\left(r_{l} / r_{c}\right)\right]} {\left[\operatorname { l n } \left\{1+\left[\left(T_{c}-T_{l}\right) / \Delta l\right] \times\right.\right.} \\
& {\left.\left[a+(b / 2)\left(T_{c}+T_{l}\right)\right]\right\}-\frac{a}{\xi} \times } \\
&\left.\ln \frac{\left(a+b T_{c}-\xi\right)\left(a+b T_{l}+\xi\right)}{\left(a+b T_{c}+\xi\right)\left(a+b T_{l}-\xi\right)}\right] .
\end{aligned}
$$

where

$$
\xi=\left\{a^{2}-2 b\left[\Delta l-(b / 2) T_{l}^{2}-a T_{l}\right]\right\}^{1 / 2}
$$

Reference to Equations [7] and [8] shows again that $\dot{m}_{F}$ is determined provided $r_{c}$ and $T_{c}$ are known. We shall show in paragraphs II-D and II-E that both $T_{c}$ and $r_{c}$ can be calculated by appropriate application of Equations [1] and [2] by utiliz-

C The experimental determination of $r_{c}$ was greatly complicated by the fact that the combustion surface was far from spherical because of severe convections currents over the droplet. 
ing the assumption that the delivery ratio of fuel to oxygen at the reaction front corresponds to the stoichiometric mixture ratio.

\section{Preliminary Remarks on the Use of Equations [1] and [2]}

One of the objectives of the present analysis is to establish an efficient method for the utilization of the basic relations given in Equations [1] and [2]. It will be convenient to restrict the analysis to spherical shells located between $r_{l}$ and $r>r_{l}$, or between $r_{c}$ and $r>r_{c}$. In this case, simple explicit expressions are obtained for the rate of energy transport by thermal conduction through the surface of radius $r$. Thus Equation [1] leads to an expression of the form

$$
4 \pi r^{2} \lambda(d T / d r)=\dot{m}_{F}\left(c_{p}\right)_{K}\left(\alpha+\beta T+\epsilon T^{2}\right) .
$$

if the specific heat is a linear function of the temperature. Here the numerical values of $\alpha, \beta$, and $\epsilon$ must be determined for each particular problem. Equation [2] becomes

$$
4 \pi r^{2}{ }_{\rho} D_{K}\left(d Y_{K} / d r\right)=\dot{m}_{F}\left(Y_{K}-\gamma_{K}\right) \ldots \ldots .
$$

where

$$
\gamma_{K}=\dot{m}_{K} / \dot{m}_{F}
$$

In the use of Equations [10] and [11], care must be taken to employ a positive value for $\gamma_{K}$ if mass flow occurs in the same direction as the fuel transport, and to use a negative value for $\gamma_{K}$ if mass flow occurs in the direction opposite to the direction of fuel transport.

It is evident by reference to Equations [9] and [10] that we can eliminate the radial distance as independent variable and write

$$
\frac{d Y_{K}}{Y_{K}-\gamma_{K}}=\chi_{K} \frac{d T}{\alpha+\beta T+\epsilon T^{2}}
$$

where the dimensionless parameter $\chi_{K}$, which is not a sensitive function of pressure and temperature, and which is assumed to be independent of $Y_{K}$, is defined by the relation

$$
\chi_{K}=\lambda / \rho D_{K}\left(c_{p}\right)_{K}
$$

A mean value is used for $\left(c_{p}\right)_{K}$ whereas $\lambda, \rho$, and $D_{K}$ are computed at any conveniently chosen temperature. Integration of Equation [12] between $Y_{o}=0$ at $r=r_{c}$ and $Y_{o}=Y_{o}$ o at $r=\infty$, corresponding to $T_{c}$ and $T_{o}$, respectively, leads directly to the value of $T_{c}$ for specified values of $T_{o}$ and $Y_{0},{ }_{0}{ }^{7}$ Similarly, integration between $Y_{F}=Y_{F, l}$ at $r=r_{l}, T=T_{l}$ and $Y_{F}=0$ at $r_{v}=r_{c}, T=T_{c}$, gives an explicit approximate expression for $Y_{F, l}^{*}=1-Y_{1, l} .^{8}$

In order to obtain the value of $r_{c}$ we can utilize Equation [9] or Equation [10]. Equation [9] can be integrated directly between $r_{c}, T_{c}$ and $\infty, T_{o}$, after expressing $\lambda$ as a linear function of $T$. Introduction of the known relation for $\dot{m}_{F}$ into the resulting expression leads to an explicit relation for $r_{c}$. The integration of Equation [10] is somewhat more involved since $\rho D_{K}$ is a linear function of $T$ to the same approximation that $\lambda$ is a linear function of $T$. Hence integration of Equation $[10]$ requires the determination of $T(r)$ prior to integration. We shall use Equation [9] for the determination of $r_{c}$.

By utilizing constant average specific heats only in the region $r>r_{c}$, it is to be expected that reasonable estimates, commensurate in accuracy with our assumed physical model, will be obtained for $T_{c}, r_{c}$, and $\dot{m}_{F}$.

\section{Determination of $T_{c}$ and of $Y_{F, \imath}$}

\section{The Reaction Zone Temperature $T_{a}$}

For the spherical shell between $r>r_{c}$ and $r_{c}$, Equation [1] becomes

7 The same result is obtained if we integrate Equation [12] from $Y_{I}=1$ at $r=r_{c}, T=T_{c}$ to $Y_{I}=Y_{I, o}=1-Y_{o}$, at $r=\infty$, $T=T_{0}$.

${ }^{8}$ The same result is obtained if we integrate Equation [12] from $Y_{I, l}$ at $r=r_{l}, T=T_{l}$ to $Y_{I}=1$ at $r=r_{c}, T=T_{c}$.
$\left.\dot{m}_{F}\left[\left(1+\gamma_{o}\right)\left(h_{P}\right)_{T}-\gamma_{0}\right)\left(h_{o}\right)_{T}\right]-\dot{m}_{F}\left[\left(1+\gamma_{O}\right)\left(h_{P}\right) T_{c}-\right.$ $\left.\gamma_{o}\left(h_{o}\right)_{T_{c}}\right]=4 \pi r^{2} \lambda(d T / d r)-\left[4 \pi r^{2} \lambda(d T / d r)\right]_{r_{c}}$.

where $h_{P}$ and $h_{o}$ denote, respectively, the specific enthalpies of products of reaction and of oxidizer. But $-\left[4 \pi r^{2} \lambda(d T /\right.$ $d r)]_{r_{c}}$ equals the total heat evolved on reaction at $T_{c}$ minus the energy transported to the fuel droplet, i.e.,

$$
-\left[4 \pi r^{2} \lambda(d T / d r)\right]_{r_{c}}=\dot{m}_{F}\left\{\begin{array}{l}
{\left[\left(1+\gamma_{o}\right)\left(h_{P}\right)_{T_{c}}+\left(h_{F}\right)_{T_{c}}+\right.} \\
\hat{\lambda}_{\left.\left.\gamma_{O}\left(h_{O}\right)_{T c}\right]-\Delta l-\left[\left(h_{F}\right)_{T_{c}}-\left(h_{F}\right)_{T l}\right]\right\}}
\end{array}\right.
$$

where $h_{F}$ is the specific enthalpy of the fuel vapor. Hence Equation [14] becomes

$$
\begin{aligned}
-4 \pi r^{2} \lambda(d T / d r)= & \dot{m}_{F}\left\{q^{*}+\gamma_{o}\left[\left(h_{o}\right)_{T}-\left(h_{o}\right)_{T *}\right]-\right. \\
& \left.\left(1+\gamma_{o}\right)\left[\left(h_{P}\right)_{T}-\left(h_{P}\right)_{T *}\right]\right\} \ldots
\end{aligned}
$$

where ${ }^{9}$

$q^{*}=-\left(1+\gamma_{o}\right)\left(h_{P}\right)_{T *}+\left(h_{F, l}\right)_{T *}+\gamma_{O}\left(h_{O}\right)_{T *}+$

$$
c_{l}\left(T_{l}-T^{*}\right)
$$

Here $T^{*}$ is a standard reference temperature (usually chosen as $298.16^{\circ} \mathrm{K}$ ), and $c_{l}$ denotes a constant specific heat for the liquid fuel in the temperature range between $T^{*}$ and $T_{l}$; the quantity $q^{*}$ differs from the standard heat of combustion for $1 \mathrm{gm}$ of liquid fuel through the addition of the term $c_{t}\left(T_{l}-\right.$ $\left.T^{*}\right)$. If $\left(c_{p}\right)_{P}$ and $\left(c_{p}\right)_{o}$ are independent of the temperature, then Equation [15] reduces to the relation

$$
\begin{array}{r}
-4 \pi r^{2} \lambda(d T / d r)=\dot{m}_{F}\left\{q^{*}+\left(T-T^{*}\right)\left\{\gamma_{o}\left(c_{p}\right)_{o}-\right.\right. \\
\left.\left.\left(1+\gamma_{o}\right)\left(c_{p}\right)_{P}\right]\right\} \ldots
\end{array}
$$

Reference to Equation [15a] shows that it is of the form

$$
4 \pi r^{2} \lambda(d T / d r)=\dot{m}_{F}\left(c_{p}\right)_{o}(\alpha+\beta T) \ldots \ldots .
$$

with

$$
\alpha=-\left[q^{*} /\left(c_{p}\right)_{o}\right]-\beta T^{*}
$$

and

$$
\beta=\left(1+\gamma_{o}\right) \hat{o}_{P}-\gamma_{o} .
$$

where $\delta_{K}=\left(c_{p}\right)_{K} /\left(c_{p}\right)_{O}$.

From Equation [10] we can obtain the appropriate expression for the mass transfer of oxidizer

$$
4 \pi r^{2}{ }_{\rho} D_{O}\left(d Y_{O} / d r\right)=\dot{m}_{F}\left(Y_{O}+\gamma_{O}\right) .
$$

where a negative sign has been used for $\gamma_{o}$ because the oxidizer flows in a direction opposite to that of the fuel. Here $D_{o}$ is the diffusion coefficient of oxidizer through the oxidizer-inert gas-combustion products mixture. Division of Equation $[15 b]$ by Equation [19] leads to the expression

$$
\frac{d Y_{O}}{Y_{O}+\gamma_{O}}=\chi_{O} \frac{d T}{\alpha+\beta T}
$$

where

$$
\chi_{O}=\lambda /\left(c_{p}\right)_{O} D_{O \rho}
$$

is a constant. Hence integration of Equation [20] between

${ }^{9}$ For the present approximate calculations the heat release $q^{*}$ and, therefore, $T_{c}, r_{c} / r_{l}$. and $\dot{m}_{F}$ are computed by neglecting the effect of dissociation of combustion products, fuel, and oxygen. The derived results would be expected to be roughly correct for gas mixtures leading to values of $T_{c}$ well below $3000^{\circ} \mathrm{K}$, i.e., for some hydrocarbon-air flames. The effect of dissociation on the calculated results can be incorporated into our present model by using an iteration procedure in which it is assumed that chemical equilibrium is established at every point. As the result of this refinement. the calculated values of $T_{c}$ and of $r_{c} / r_{l}$ are decreased by a first iteration; the temperature profile toward the oxidizer is flattened and the temperature raised because the reassociating gases act as a distributed heat source on recombination. The diffusive flow of oxygen inward is then increased, thereby leading to a further decrease of $r_{c} / r_{l}$. Rough calculations show that the net effect of dissociation on the calculated values of $\dot{m}_{F}$ for benzene-air flames is probably less than 10 per cent, although both $T_{c}$ and $r_{c} / r_{l}$ are decreased appreciably. 
$Y_{o}=0, T=T_{c}$ and $Y_{O}=Y_{o},{ }^{\circ}, T_{o}$ leads to the following explicit relation for $T_{\text {c }}$

$$
T_{c}=\frac{\beta T_{o}-\alpha\left\{\left[1+\left(Y_{o, o} / \gamma_{o}\right)\right]^{(\beta / \chi o)}-1\right\}}{\beta\left[1+Y_{o, o / \gamma o}\right]^{(\beta / \chi o)}} \ldots
$$

\section{The Weight Fraction Profile of Fuel Vapor}

It is evident from the formulation of the present problem that $Y_{F}$ cannot equal unity at the droplet surface. For this reason it is of interest to estimate $Y_{F}$ at $r_{l}$ approximately.

From Equation [3a] and Equation [10], written in terms of the fuel, it follows that

$$
\frac{d Y_{F}}{Y_{F}-1}=\frac{\lambda}{\rho D_{F}} \frac{d T}{(b / 2) T^{2}+a T+\left[\Delta l-a T_{l}-(b / 2) T_{l}^{2}\right]}
$$

where $\lambda / \rho D_{F}$ is independent of temperature and is assumed to be independent also of $Y_{F}$. Integration of Equation [22] between $Y_{F}=Y_{F, l}$ at $T=T_{l}$ and $Y_{F}=0$ at $T=T_{c}$ leads to the result

$$
Y_{F, l}^{\prime}=1-{\frac{\left(a+b T_{c}-\xi\right)\left(a+b T_{l}+\xi\right)}{\left(a+b T_{c}+\xi\right)\left(a+b T_{l}-\xi\right)}}^{-\left(\lambda / \rho D_{F \xi}\right)}
$$

where $\xi$ has been defined in Equation [8].

\section{E Determination of the Combustion Radius}

Equation [15b], which was derived on the assumption that the specific heats of oxidizer and inert gas are constant for $r>r_{c}$, may be used directly for the determination of $r_{c}$. After replacing $\lambda$ by $\lambda_{l}\left(T / T_{l}\right)$ and integrating from $r_{c}, T_{c}$ to $\infty, T_{s}$, the following relation is obtained

$$
\frac{1}{c}=\frac{4 \pi \lambda_{l}}{m_{F}\left(c_{p}\right)_{o} T_{l}}\left[\frac{1}{\beta}\left(T_{o}-T_{c}\right)-\frac{\alpha}{\beta^{2}} \ln \frac{\alpha+\beta T_{o}}{\alpha+\beta T_{c}}\right] .
$$

where $\alpha$ and $\beta$ are defined in Equations [16] and [17], respectively.

From Equations [7] and [24] we can now obtain an explicit expression for $r_{c} / r_{l}$. Thus we may write Equation [7] in the form

$$
\dot{m}_{F}=\frac{4 \pi \lambda_{l} r_{l}}{b T_{l}\left[1-r_{l} / r_{c}\right]} \Phi \ldots \ldots \ldots \ldots
$$

where

$$
\begin{array}{r}
\Phi=\ln \left\{1+\left[\left(T_{c}-T_{l}\right) / \Delta l\right]\left[a+(b / 2)\left(T_{c}+T_{l}\right)\right]\right\}- \\
\frac{a}{\xi} \ln \frac{\left(a+b T_{c}-\xi\right)\left(a+b T_{l}+\xi\right)}{\left(a+b T_{c}+\xi\right)\left(a+b T_{l}-\xi\right)}
\end{array}
$$

From Equations [24] and [7a] it is seen that

$$
\frac{r_{c}}{r_{l}}=1+\frac{\left(c_{p}\right)_{o} \Phi}{b}\left[\frac{1}{\beta}\left(T_{o}-T_{c}\right)-\frac{\alpha}{\beta^{2}} \ln \frac{\alpha+\beta T_{o}}{\alpha+\beta T_{c}}\right]^{-1} \ldots
$$

Reference to Equation [26] shows the interesting result, which is in accord with some of the experimental observations, that $r_{c} / r_{l}$ is a constant for fixed values of the physicochemical parameters. Hence Equation [7a] shows that $\dot{m}_{F}$ is a linear function of $r_{l}$.

The linear functional relation between $\dot{m}_{F}$ and $r_{l}$ has been used by Godsave $(3,5)$ to obtain the following expression for the variation of droplet diameter with time

$$
d^{2}=d_{0}^{2}-K^{\prime} t
$$

Here $d$ is the droplet diameter at time $t, d_{0}$ is the initial droplet diameter, and the evaporation constant $K^{\prime}$ is defined by the relation

$$
K^{\prime}=2 \dot{m}_{F} / \pi r_{t} \rho_{\imath} \ldots \ldots \ldots \ldots \ldots \ldots \ldots \ldots \ldots \ldots \ldots
$$

Since $K^{\prime}$ is independent of $r_{l}$, it is a convenient parameter for comparing burning rates of different fuels for arbitrary droplet sizes.
III Comparison Between Calculated and Observed Results for the Burning of Single Droplets of Fuel

We have obtained in the preceding Section II a complete description for the burning of single droplets of fuel in an oxidizing atmosphere according to a simplified model. It is the purpose of the present discussion to compare calculated and observed values for $r_{c} / r_{l}$ and for $K^{\prime}=2 \dot{m}_{F} / \pi r_{l} \rho_{l}$.

The procedure for calculating various quantities, including $r_{v} / r_{l}$ and $K^{\prime}$, involves the following steps: (a) For suitably chosen values of the physicochemical parameters we obtain $T_{c}$ from Equation [21]. (b) The limiting weight fraction $Y_{F, l}$ is next obtained from Equation [23]. (c) The quantity $r_{c} / r_{l}$ is given by Equation [26]. (d) Finally $2 \dot{m}_{F} / \pi r_{l} \rho_{l}$ is calculated either from Equation [7] or from Equation [7a].

For the sake of completeness we have determined also the $T$ vs. $r$ and $Y_{K}$ vs. $r$ profiles for the burning of a benzene droplet in air if $T_{o}=300^{\circ} K, r_{l}=0.005 \mathrm{~cm}$. The results of these calculations have been considered previously and are given in Figs. 2a to 2 d.

\section{A Summary of Calculated Results}

The results of calculations for the burning of benzene, ethyl alcohol, ethyl benzene, $n$-heptane, and toluene in air are summarized in Table 1. Also listed in Table 1 are appropriate values of the physicochemical parameters, which were for the most part taken from Godsave's papers $(3,5)$.

\section{B Comparison of Calculated and Observed Evaporation Constants}

The calculated and observed evaporation constants are contrasted in Table 2 . Reference to Table 2 shows satisfactory agreement between calculated and observed values of $K^{\prime}$ (and hence of $\dot{m}_{F^{\prime}}$ ). This result suggests that the physical model upon which the present analysis of burning of fuel drops is based represents a useful first approximation for the compounds considered. Unfortunately, experimental data are available only for fuels with very similar values of $K^{\prime}$. For this reason the comparison between theory and experiment is not as stringent a test of the theory as might be desired. In particular, there are serious doubts that the basic physical assumptions involved in our model are applicable, for example, to the burning of an aniline droplet in nitrogen dioxide or in nitric acid vapors. Experimental studies on systems of this type will be carried out in our laboratory in the near future and compared with the results of the theoretical calculations.

TABLE 2 COMPARISON OF CALCULATED AND OBSERVED VALUES FOR THE EVAPORATION CONSTAN'T $K^{\prime}$

$\begin{array}{lcc}\text { Compound } & \begin{array}{c}\text { of } K^{\prime}, \\ \mathrm{cm}^{2} / \mathrm{sec}\end{array} & \begin{array}{c}\text { of } K^{\prime}, \\ \mathrm{cm}^{2} / \mathrm{sec}\end{array} \\ \text { Benzene } & 0.0097 & 0.0100 \\ \text { Ethyl alcohol } & 0.0081 & 0.0079 \\ \text { Ethyl benzene } & 0.0086 & 0.0085 \\ n \text {-heptane } & 0.0097 & 0.0086 \\ \text { Toluene } & (0.0066) & 0.0087\end{array}$

We have recently carried out some theoretical calculations on the evaporation rate of pure compounds into still air by replacing $T_{c}$ and $T_{l}$ in Equation [4] by the known ambient and droplet temperatures, respectively. The results obtained for water droplets, on the assumption that $1 / r_{c} \ll 1 / r_{l}$, are in very satisfactory agreement with empirically determined values. 
TABLE 1 PHYSICOCHEMICAL PARAMETERS AND CALCULATED VALUES FOR THE BURNING OF DROPS OF BENZENE, ETHYL ALCOHOL, ETHYL BENZENE, $n$-HEPTANE, AND TOLUENE IN AIR AT ATMOSPHERIC PRESSURE

$\quad$ FuEL
Benzene
Ethyl alcohol
Ethyl benzene
n-Heptane
Toluene

\begin{tabular}{|c|c|c|c|c|}
\hline$T_{\boldsymbol{o}},{ }^{\circ} \mathbf{K}$ & 6. 0 & $\gamma o$ & $T_{l,}{ }^{\circ} \mathbf{\mathbf { K }}$ & $\rho_{l}, \mathrm{GM} / \mathrm{CM}^{3}$ \\
\hline 300 & 0.23 & 3.08 & 353 & 0.84 \\
\hline 300 & 0.23 & 2.09 & 351 & 0.79 \\
\hline 300 & 0.23 & 3.07 & 409 & 0.84 \\
\hline 300 & 0.23 & 3.52 & 371 & 0.68 \\
\hline 300 & 0.23 & 3.13 & 384 & 0.84 \\
\hline \multicolumn{2}{|c|}{$\begin{array}{c}\left(\boldsymbol{\Theta}_{p}\right)_{O}, \\
\mathrm{CAL} / \mathrm{GM}^{\circ} \mathrm{K}\end{array}$} & $\delta_{P}$ & $\begin{array}{l}T^{*}, \\
{ }^{*} \\
\mathbf{K}\end{array}$ & $\begin{array}{c}q^{*},{ }^{10} \\
\mathrm{CAL}_{\mathrm{AL}} / \mathrm{GM}\end{array}$ \\
\hline \multicolumn{2}{|c|}{0.26} & 1.35 & 298 & 9,790 \\
\hline \multicolumn{2}{|c|}{0.26} & 1.58 & 298 & 6,460 \\
\hline \multicolumn{2}{|c|}{0.26} & 1.35 & 298 & 9,850 \\
\hline \multicolumn{2}{|c|}{0.26} & 1.58 & 298 & 10,630 \\
\hline \multicolumn{2}{|c|}{0.26} & 1.39 & 298 & 9,750 \\
\hline
\end{tabular}

$\Delta l, \mathrm{CAL} / \mathrm{GM}$
94.0
204
81.0
75.6
87.0

$a, \mathrm{CAL} / \mathrm{GM}^{\circ}{ }^{\circ} \mathrm{K}$
0.34
0.44
0.40
0.44
0.37

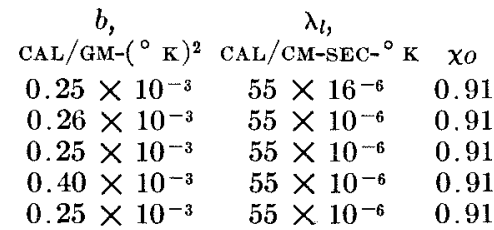

FUEL

Benzene

Ethyl alcohol

Ethyl benzene

$n$-heptane

0.26

1.39298

\begin{tabular}{|c|c|c|c|c|c|}
\hline$\beta$ & $\alpha,^{\circ} \mathbf{K}$ & $\begin{array}{l}T_{c},{ }^{10} \\
{ }_{K}\end{array}$ & $\begin{array}{c}\xi, \\
\mathrm{CAL} / \mathrm{GM}^{-}{ }^{\circ} \mathrm{K}\end{array}$ & $\Phi$ & $r_{c} / r^{10}$ \\
\hline 2.43 & $-38,400$ & 3450 & 0.37 & 1.51 & 9.6 \\
\hline 2.79 & $-25,700$ & 3100 & 0.42 & 1.05 & 5.3 \\
\hline 2.43 & $-38,600$ & 3470 & 0.46 & 1.48 & 9.3 \\
\hline 3.63 & $-42,200$ & 3230 & 0.54 & 1.75 & 8.6 \\
\hline 2.61 & $-38,300$ & 3370 & 0.42 & 1.43 & 9.5 \\
\hline
\end{tabular}

$K^{\prime},{ }^{10}$ $\mathrm{CM}^{2} / \mathrm{SEC}$

$10.0 \times 10^{-3}$

$7.9 \times 10^{-3}$

$8.5 \times 10^{-3}$

$8.6 \times 10^{-3}$

${ }^{10}$ See footnote 9 for a discussion of the effect of dissociation on $T_{c}$ and on $r_{c} / r_{l}$. For benzene $T_{c}$ is decreased to about $2600^{\circ} \mathrm{K}$ when proper allowance is made for dissociation. 'The effect of dissociation on $K^{\prime}$ is probably small.

\section{Comparison of Observed and Calculated Values of $r_{c} / r_{l}$}

Our theoretical analysis is based on the assumption that a spherical reaction surface surrounds the burning droplet. On the other hand, photographs of burning droplets indicate that the luminous region is of the form shown in Fig. 3. Godsave measured the diameter of the sphere corresponding to the dotted circle in Fig. 3 and stated that the ratio of this diameter to the droplet diameter is a constant, characteristic for the fuel, when burning in air.

The values of $r_{c} / r_{l}$ calculated from Equation [26] are contrasted in Table 3 with the values of $r_{c} / r_{l}$ deduced by Godsave from his photographs of burning fuel drops. Reference to Table 3 shows that the calculated values are appreciably larger than the observed data. There are several obvious reasons for the observed discrepancies. Thus reference to Fig. 3 shows that the "still" droplet was actually subjected to strong convection currents during burning; the value of $r_{c} / r_{l}$ for a spherical surface with area equivalent to the area of the observed luminous zone is roughly double that of Godsave's tabulated values. Furthermore, it is not evident that the reaction surface in our idealized model should, in fact, be identified with the region of maximum luminosity. The surface for maximum temperature gradients, as determined from schlieren photographs (5), corresponds to larger "observed" values of $r_{c} / r_{l}$ than are listed in Table 3 .

$\begin{array}{ccc}\text { TABle } 3 & \begin{array}{c}\text { COMParison of Calculated and "OBserved" } \\ \text { Combustion Radi }\end{array} \\ & \text { "Observed" values } & \text { Calculated values } \\ \text { Fuel } & \text { of } r_{c} / r_{l} & \text { of } r_{c} / r_{l} \\ \text { Benzene } & 2.97 & 9.6 \\ n \text {-heptane } & 3.03 & 8.5 \\ \text { Toluene } & 2.59 & 9.5\end{array}$

The discrepancies between calculated and "measured" values of $r_{c} / r_{l}$ emphasize also the need for refinement in our theoretical description of the burning process. Thus it is apparent that the introduction of a spherical reaction shell of finite thickness will lead to lower values of $T_{\mathrm{c}}$ and also to lower effective values of $r_{c} / r_{l}$; as indicated in footnote 9 , a similar effect is produced also if proper allowance is made for dissociation by assuming that chemical equilibrium is established at every point. Presumably the changes in $T_{c}$ and in $r_{c} / r_{l}$ will largely compensate for each other in the calculation of $K^{\prime}$ or $\dot{m}_{F}$, thereby accounting for the satisfactory agreement between calculated and observed values of $\dot{m}_{F}$.
In conclusion it seems appropriate to note that some experimental evidence exists which is not in accord with the idea that $r_{c} / r_{l}$ is constant. Thus Hall and Diederichsen (10) state that their studies of the burning of single drops of fuel suggest that the distance between the flame front and the drop surface remains constant.

\section{Effect of Pressure on the Burning Process}

The results of the present simplified analysis indicate that the only dependence of the burning rate on pressure occurs through the variation of the boiling points and latent heats of evaporation with pressure. The values of $T_{c}$ and $r_{c} / r_{l}$ are essentially independent of pressure. The results of Hall and Diederichsen show that the burning rate is roughly proportional to the one-fourth power of the pressure (10). Although the present analysis does not lead to this simple functional relation, detailed calculations show that the burning rates, as predicted by our theory, increase with pressure, primarily because of the decrease of latent heat of evaporation at the higher boiling temperatures associated with the increased pressure. Again it is clear that a quantitative description of the dependence of $K^{\prime}$ or $\dot{m}_{F}$ on pressure requires extension of the present analysis in several respects. Thus chemical reaction rates are sensitive functions of the pressure (for a

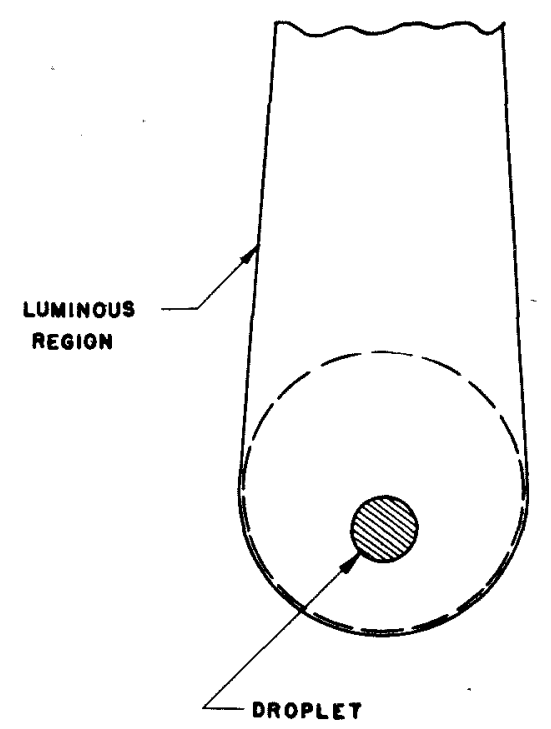

FIG. 3 SChEMATIC DIAGRAM OF FLAME FRONT SURROUNDING A BURNING DROPLET 
"complicated process, an over-all dependence of the reaction rate on the square of the pressure is not unreasonable) and, therefore, a very weak dependence of $\dot{m}_{F}$ on reaction rates could account for the observed variation of $\dot{m}_{F}$ with pressure. Furthermore, the effects of radiant heat transfer to the droplet surface, which have been neglected in the present study, increase rapidly with pressure and could account for a weak dependence of $\dot{m}_{F}$ on pressure. Finally, the role of the inevitable convection currents requires quantitative study and may well lead to a slight variation of $\dot{m}_{F}$ with pressure.

\section{References}

1 "The Combustion of Drops in a Fuel Spray," by G. A. E. Godsave, National Gas Turbine Establishment (England) Memorandum No. M. 95, 1950.

"One-Dimensional Velocity Variation of a Burning Droplet," by C. C. Miesse, Heat Transfer and Fluid Mechanies Institute, Los Angeles, 1953.

"Studies on the Spontaneous Ignition of Fuels Injected in a Hot Air Stream," by B. P. Mullins; Part I, National Gas Turbine Establishment (England) Report No. R. 89, 1951; Part II, National Gas Turbine Establishment (England) Report No. R. 90,1951 .

2 "The Burning of Single Drops of Fuel: Part I, Temperature Distribution and Heat Transfer in the Pre-Flame Region," by G. A. E. Godsave, National Gas Turbine Establishment (England) Report No. R. 66, 1950.
3 "The Burning of Single Drops of Fuel: Part II, Experimental Results," by G. A. E. Godsave, National (ias Turbine Establishment (England) Report No. R. 87, 1951.

4 "An Experimental Study of the Evaporation and Combustion of Falling Droplets," by J. E. C. Topps, Journal of the Institute of Petroleum, vol. 37, 1951, pp. 535-537.

5 "The Burning of Single Drops of Fuel: Part III, Comparison of Experimental and Theoretical Burning Rates and Discussion of the Mechanism of the Combustion Process," by G. A. E. Godsave, National Gas Turbine Establishment (England) Report No. R. 88, 1952.

6 "The Combustion of Liquid Fuel in a Gas Stream," by D. B. Spalding, Part I, Fuel, vol. xxix, 1950, pp. 2-7; Part II, Fuel, vol. xxix, 1950, pp. 25-32.

7 "On Maximum Evaporation Rates of Liquid Droplets in Rocket Motors," by S. S. Penner, Journal of the American Rocket Society, vol. 23, 1953, pp. 85-88.

"Maximum Evaporation Rates for Non-Isothermal Droplets," by F. W. Hartwig, Journal of the American Rocket Society, vol. 23,1953 , pp. 242-243.

8 Sorbonne Lectures, by 'Th. von Kármán, Paris, 1952-1953.

9 "Handbook of Chemistry and Physies," Chemical Rubber Publishing Co., Cleveland, 33rd ed., 1951, p. 1579

10 "An Experimental Study of the Burning of Single Drops of Fuel in Air at Pressures up to Twenty Atmospheres," by A. R. Hall and J. Diederichsen, Fourth (International) Symposium on Combustion, Williams and Wilkins Company, Baltimore, 1953, pp. 837-846. 